\title{
METALLOSUPRAMOLECULAR CHEMISTRY WHAT IS IT?
}

\author{
Peter J. Steel \\ Department of Chemistry, \\ University of Canterbury, Christchurch New Zealand.
}

The term supramolecular chemistry was first introduced by Nobel laureate Jean-Marie Lehn, in 1978, to describe the "chemistry of molecular assemblies". Whereas traditional chemistry involves the study of molecules consisting of atoms held together by strong covalent bonds, supramolecular chemistry uses molecules as the basic building blocks for the construction of larger aggregates. These are held together by weak interactions, such as hydrogen bonds, electrostatic interactions, van der Waals interactions, or the stacking of aromatic rings. Although these interactions are individually much weaker than the covalent bonds in organic molecules, by employing large numbers of them very robust assemblies of molecules can be formed. In a similar way, nature uses weak bonds to hold together the strands of DNA, protein assemblies, and the majority of cellular machinery.

One of the most versatile ways to build such assemblies is to make use of interactions between metal ions (M) and donor groups in organic molecules (ligands, L), as has long been exploited in traditional coordination chemistry. By employing ligands that bridge more than one metal centre it is possible to construct one-, two- or threedimensional architectures, based on M-L interactions. ${ }^{1}$ This is metallosupramolecular chemistry, a term introduced by Constable in 1994, wherein the metals act as a type of "glue" to hold together assemblies of organic molecules. The magnitude of such $\mathrm{M}-\mathrm{L}$ interactions varies from very weak to very strong, depending on the nature of $\mathrm{M}$ and L.

The beauty of this chemistry lies not only in the diverse range of supramolecular topologies possible, but in the simplicity of their preparation. Traditional organic syntheses involve the stepwise construction of molecules through sequences of chemical reactions, frequently requiring tedious manipulation of the various functional groups, and often in low overall yields. In contrast, metallosupramolecular syntheses utilise self-assembly processes, in which simply mixing together metal and ligand building blocks yields the desired product, in a single process in high yield. This spontaneous self-organisation of a number of molecular components into a single aggregated structure occurs through molecular 
recognition, in which structural information encoded into the precursors leads to their self-assembly. Through reversible processes that explore all the possible structures, the single most stable product is obtained.

Thus, the building blocks for a metallosupramolecular synthesis are simply metal ions and bridging organic ligands. Control over the self-assembly process is based on the coordination requirements of the metals employed and the relative positions of the donor atoms in the bridging ligands. Figure 1 shows a selection of metals with different coordination numbers and geometries, along with a few commonly employed bridging ligands.

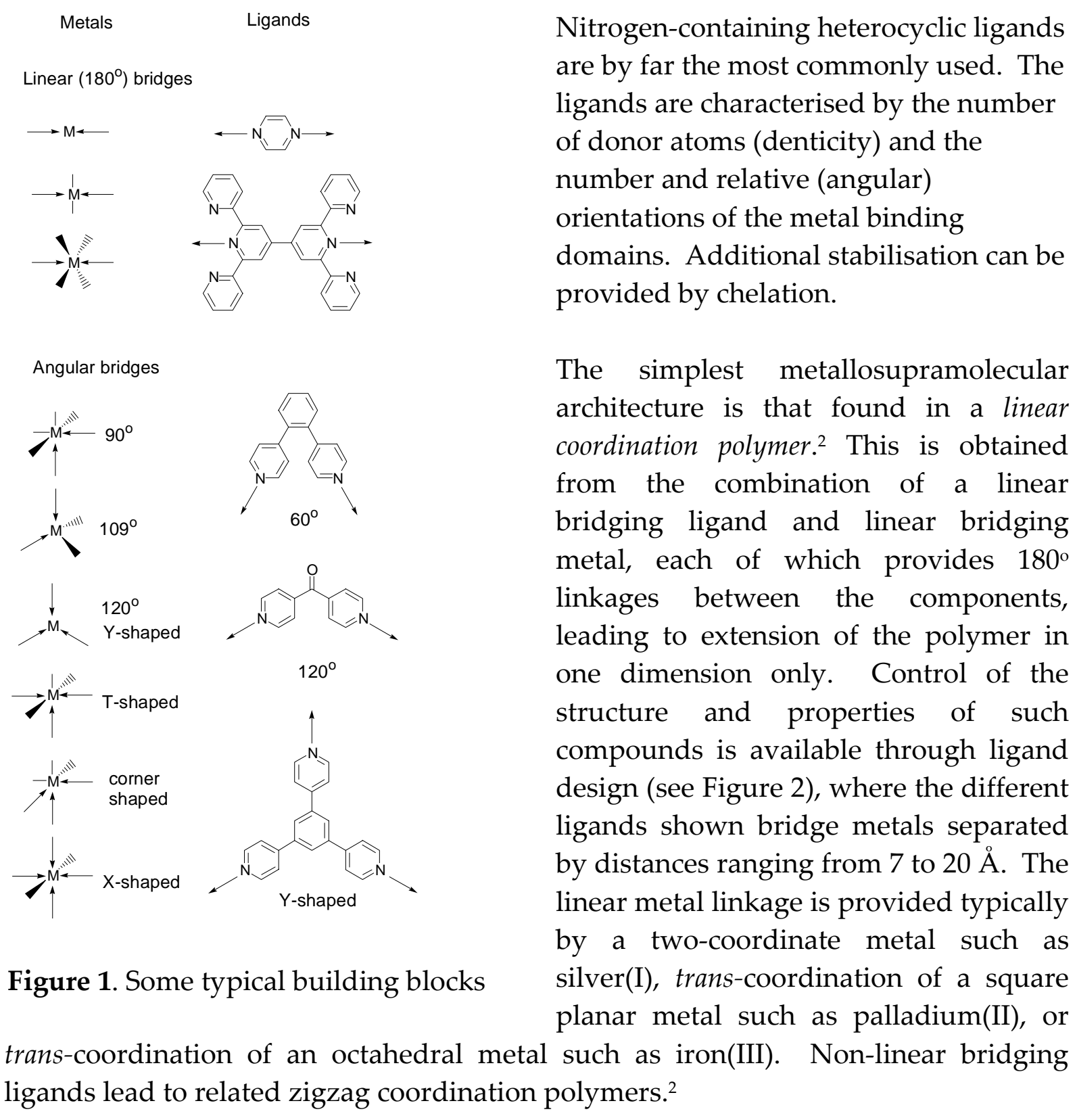




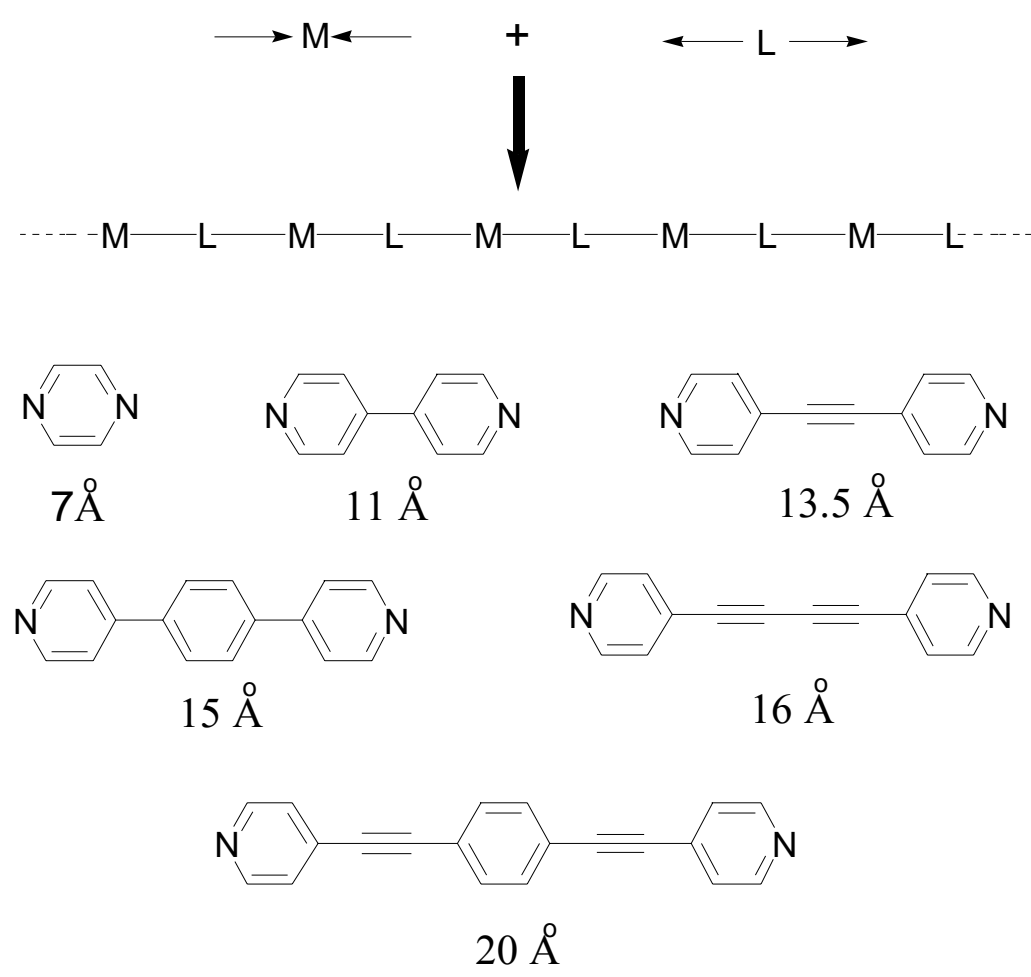

Figure 2.. Coordination polymers and M-M separations.

Two-dimensional polygons are popular targets for metallosupramolecular synthesis. The simplest of these are molecular squares.

Since a square consists of four sides and four corners, these can be easily assembled from the combination of a linear bridging ligand and a metal that provides a $90^{\circ}$ bridge, e.g. cis coordination of a square planar or octahedral metal. The first example of these was reported by Fujita ${ }^{3}$ using 4,4'-bipyridine for the sides and Pd(en) for the corners (Figure 3, M = Pd).

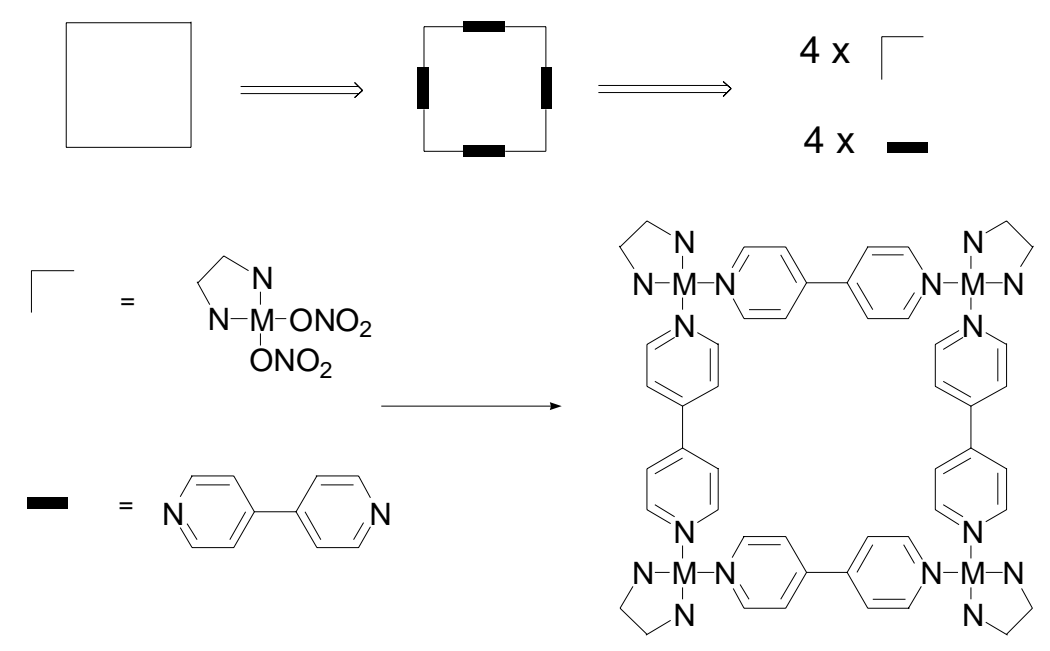

Figure 3. A molecular square. 
Such a species is favoured over a zigzag coordination polymer on thermodynamic grounds. One can control the size of such squares simply by varying the length of the bridging ligand. Less symmetrical molecular rectangles require the use of two bridges of different lengths.

Other symmetrical polygons (Figure 4, below) are available using a similar approach. ${ }^{4}$ Molecular hexagons require $120^{\circ}$ angular bridges, which can be either trigonally coordinated metals, or, more commonly, ligands based on an $\mathrm{sp}^{2}$ hybridised carbon (such as a ketone) to provide the required angle. Pentagons require a $108^{\circ}$ corner, which is conveniently approximated by the tetrahedral angle. Triangles provide an interesting challenge $\mathrm{e}^{5}$ as a metal cannot routinely supply the requisite $60^{\circ}$ angular component, and hence an angular bridging ligand is used in combination with a linear metallic bridge to generate the desired geometry.

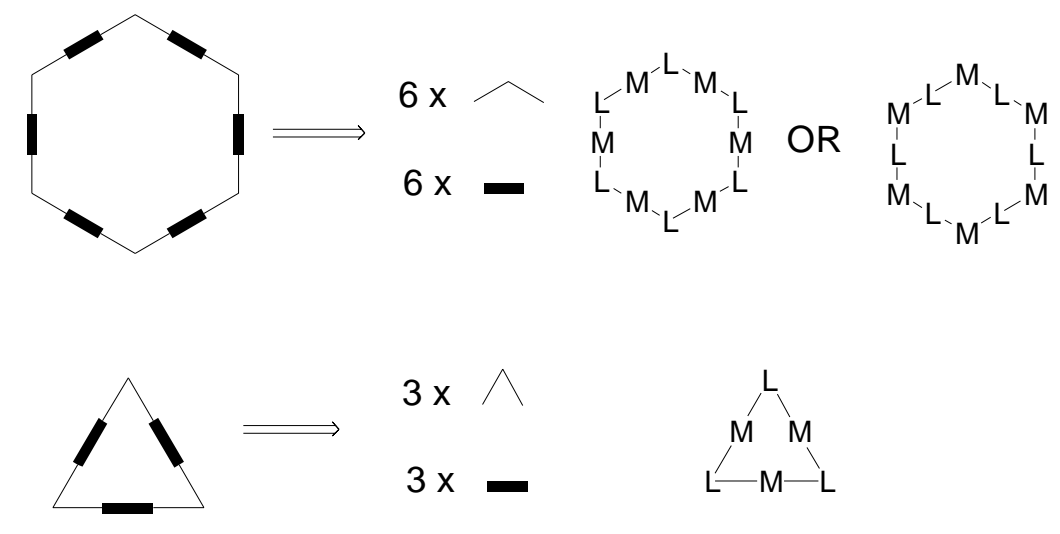

Figure 4. Molecular hexagons and triangles.

Molecular squares can be combined to form species of higher dimensionality. Figure 5 shows the schematic retro-synthesis of a molecular window. This requires twelve linear components, four $90^{\circ}$ corners, four T-shaped linkages, and a central X-shaped bridge. In a spectacular example of self-assembly, Drain ${ }^{6}$ reported the preparation of such a species using trans- $\mathrm{PdCl}_{2}$ as the linear bridge and three different porphyrinbased ligands as the three angular components.

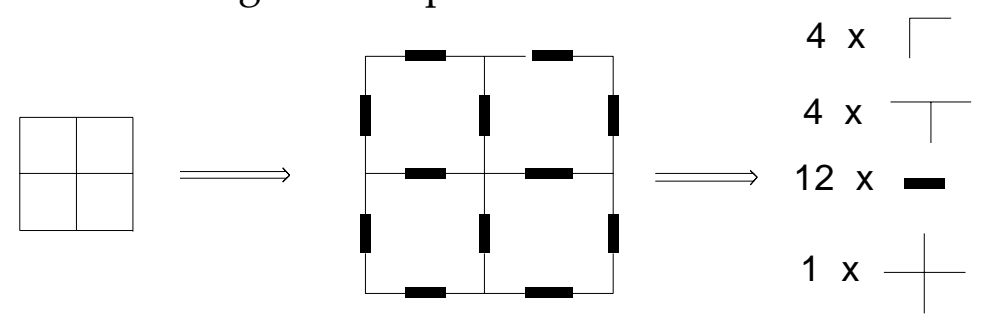

Figure 5. A molecular window. 
Squares can be assembled into one-dimensional polymeric aggregates. When joined at their corners this leads to molecular lattices. Figure 6 below shows how these consist of $90^{\circ}$ angular bridges linked by X-shaped components, in a 2:1 ratio, respectively. As is often the case in metallosupramolecular chemistry, two synthetic approaches to such compounds are possible: the metal can be used as the rightangular bridge with an organic ligand providing the $\mathrm{X}$-shaped component (Figure 6a) or vice versa (Figure 6b). Similarly, squares can be joined at the sides to form molecular ladders, which comprise linear components as rungs and sides linked together by T-shaped components, this time in a 3:2 ratio. Once again, the organic ligand can be either the linear bridge (Figure 6c) or, less commonly, the T-shaped component (Figure 6d). This concept can extended into the second dimension to form a two-dimensional square tile pattern which consists of a 1:1 ratio of linear bridges (usually the organic ligand) and X-shaped components (Figure 6e). Logically, the size of this tiling system is controlled by the lengths of the sides, which is readily controlled by means of ligand design. ${ }^{7}$

\section{$\times \times \times$}

(a)

(a) $\quad M^{M} M^{M} M$

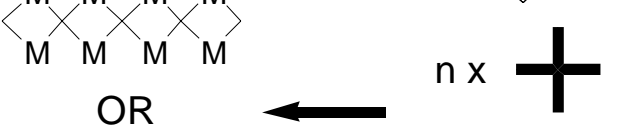

(b)
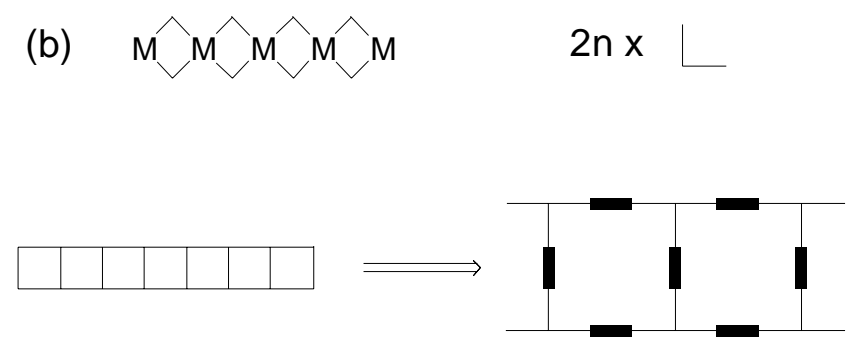

$M L M L M L M$

(c)

(d)
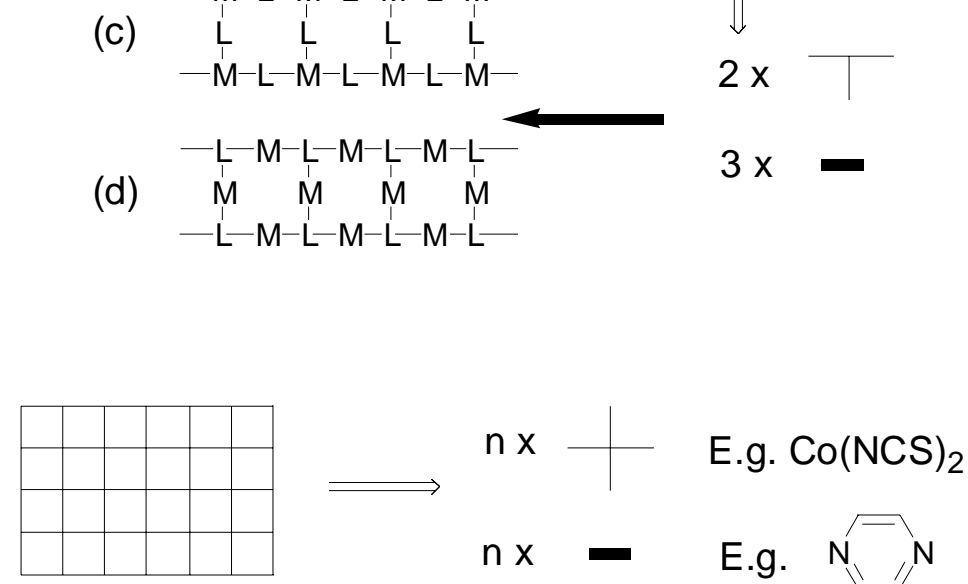

(e)

Figure. 6. Molecular lattices, ladders and square tiles. 
The examples given so far all represent zero, one- or two-dimensional architectures. The principles involved can further be extended into three-dimensions. For example, molecular cubes require eight corners and twelve linear edges (Figure 7).

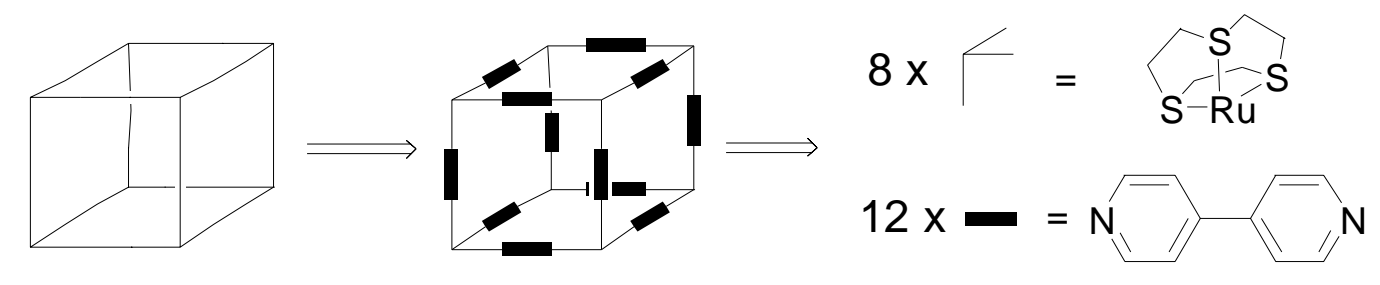

Figure 7. A molecular cube.

The first such cube was assembled using $4,4^{\prime}$-bipyridine as the linear bridge with facially coordinated ruthenium atoms to provide the corners. ${ }^{8}$ Inversely, a cube has been constructed using an organic ligand as the corners and linear two-coordinate silver atoms as the edges. ${ }^{9}$

Because adamantine, $\mathrm{C}_{10} \mathrm{H}_{16}$, is the most stable and simplest three-dimensional hydrocarbon, octahedral adamantanoid cages (Figure 8) represent the most readily accessible symmetrical three-dimensional structures. These have the same symmetry and topology as adamantane and are assembled from ten components, namely six two-connector groups and four three-connector groups. Of the two possible approaches to such entities, the $\mathrm{M}_{6} \mathrm{~L}_{4}$ option is the more common. The earliest of these used palladium as the two-connector component, either in a cis-10 or trans ${ }^{-11}$ coordination mode. In an intriguing series of experiments, Fujita ${ }^{12}$ has described the use of one such cage as a molecular-scale flask in which chemical reactions were carried out in the internal cavity of the cage and the products confined therein. Raymond has used the inverse approach to prepare various $\mathrm{M}_{4} \mathrm{~L}_{6}$ cages, one of which has recently been shown to exhibit interesting memory effects. ${ }^{13}$

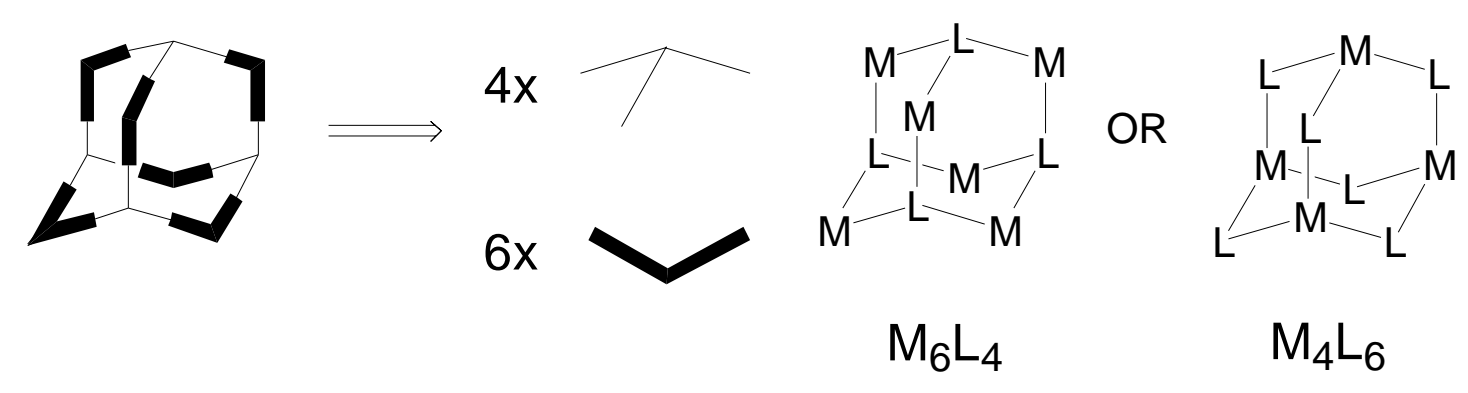

Figure 8. Adamantanoid cages.

In one of the most remarkable reactions ever reported, Stang described the formation of a metallosupramolecular dodecahedron. ${ }^{14}$ This Platonic solid consists of thirty edges and twenty vertices. Simply by combining a 3:2 ratio of an organometallic linear bridge with a tripodal Y-shaped ligand, a dodecahedral structure self-assembled 
from the fifty components with the formation of sixty M-L bonds (Figure 9) — and all this happened in quantitative yield! Contrast this with the first synthesis of the corresponding hydrocarbon dodecahedrane, $\mathrm{C}_{20} \mathrm{H}_{20}$, which took twenty years, twenty three laborious steps and gave an overall yield of $<0.1 \% .^{15}$

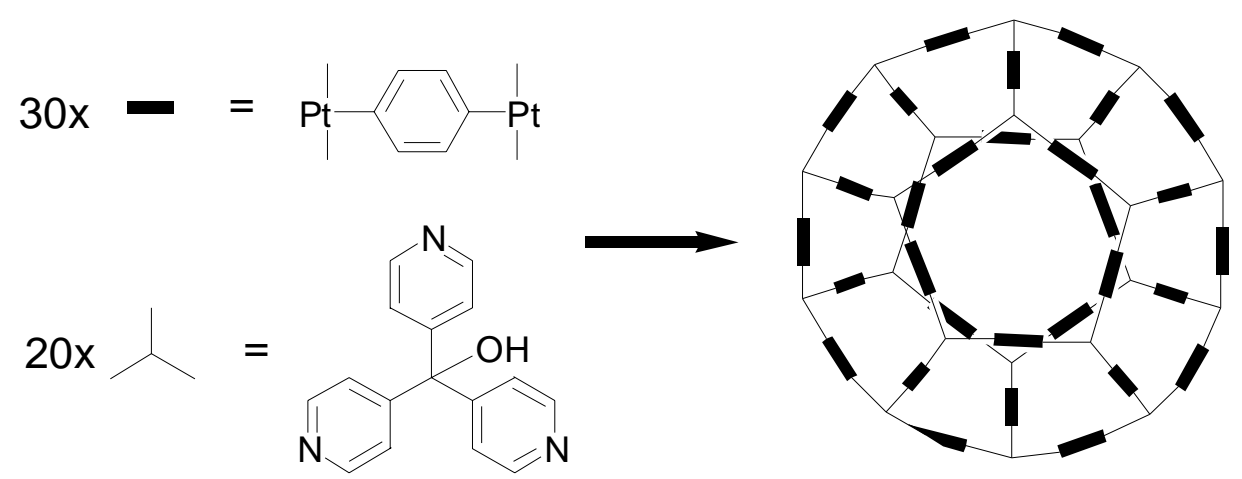

Figure 9. Self-assembly of a dodecahedral cage.

Among the most popular topologies studied in this area are helicates, which represent some of the earliest examples of rationally designed metallosupramolecular species. ${ }^{16}$ These are obtained by wrapping strands of organic ligands around metals in a helical fashion (see Figure 10).

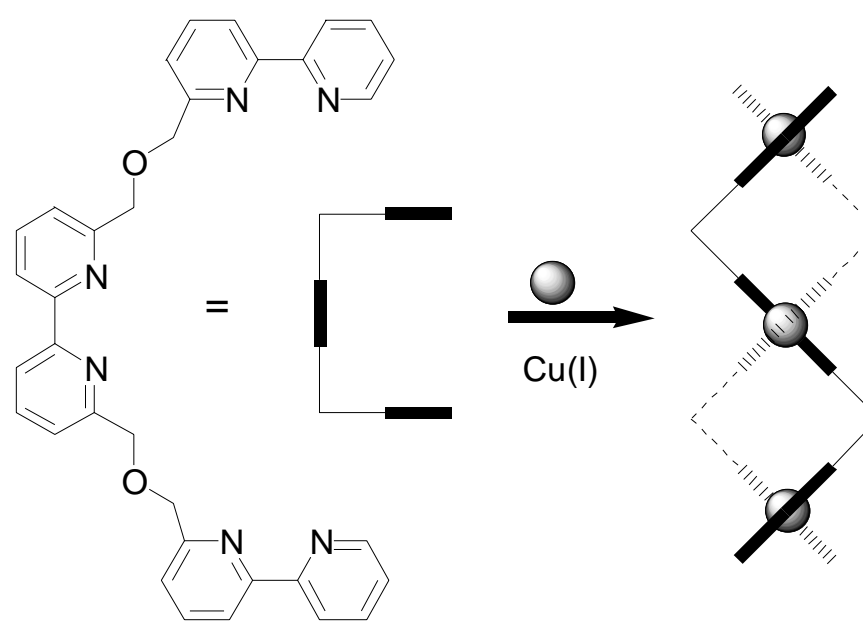

Figure 10. Self-assembly of a trinuclear double helicate.

Early examples by Lehn and by Constable used ligands containing multiple pyridine rings to prepare double-stranded helicates containing up to five metals. In one notable experiment, Lehn described how a mixture of four different ligands was reacted with copper(I) ions to produce four discrete double helicates, rather than a complex mixture of products. This is a concept that has recently been referred to as 
self-sorting, ${ }^{17}$ wherein complex mixtures of compounds are able to use molecular recognition principles to sort themselves into self-complementary arrays. Triplestranded helicates have since become well-studied ${ }^{16}$ and, more recently, a quadruplestranded helicate reported. ${ }^{18}$ Circular helicates are also known. ${ }^{16}$

Another area of intense interest has been that of catenates. Catenanes are molecules that consist of two or more interlocked rings. These rings are not chemically bonded, but cannot be separated without cleaving one of the rings. The earliest catenates used metal ions as templates to hold together acyclic precursors suitably oriented for the formation of interlocked rings (Figure 11). ${ }^{19}$ More recently, Fujita has described the self-assembly of catenates by utilising stacking interactions between aromatic rings in polar media as an external driving force for their formation. ${ }^{20}$

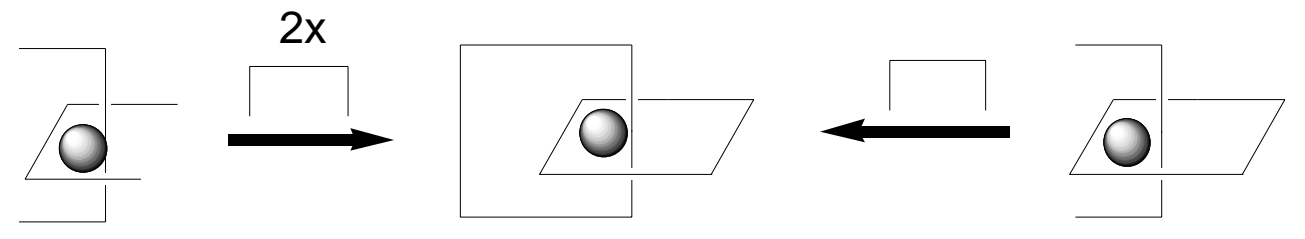

Figure 11. Template syntheses of catenates.

The examples discussed above represent just a few of the many fascinating supramolecular architectures that have been reported over the past decade or so. No doubt many other even more interesting structures are yet to be described, perhaps by replacing the rigid synthons described above with more flexible $\mathrm{M}$ and $\mathrm{L}$ components, so as to provide access to structures not available to the rigid precursors. It remains to be seen whether the many claims that these metallosupramolecular species will find useful applications in the highly topical areas of material science and nanotechnology will be fulfilled.

\section{References}

1. For recent reviews see: Leininger, S., Olenyuk, B., and Stang, P. J., Chem. Rev., 2000, 100, 853-908; Swiegers, G. F. and Malefetse, T. J., Chem. Rev., 2000, 100, 3483; Moulton, B. and Zaworotko, M. J., Chem. Rev., 2001, 101, 1629; Fujita, M., Umemoto, K., Yoshizawa, M., Fujita, N., Kusukawa, T., and Biradha, K., J. Chem Soc., Chem. Commun., 2001, 509; Johnson, D. W. and Raymond, K. N., Supramol. Chem., 2001, 13, 639; Holliday, B. J. and Mirkin, C. A., Angew. Chem. Int. Ed., 2001, 40, 2022; Swiegers, G. F. and Malefetse, T. J., Coord. Chem. Rev., 2002, 225, 91.

2. Khlobystov, A. N., Blake, A. J., Champness, N. R., Lemenovskii, D. A., Majouga, A. G.: Zyk, N. V., and Schroder, M., Coord. Chem. Rev., 2001, 222, 155.

3. Fujita, M., Yakazi, J., and Ogura, K., J. Am. Chem. Soc., 1990, 112, 5645.

4. Stang, P. J., Chem. Eur. J., 1998, 4, 19. 
5. Steel, P. J. and Webb, N. C., Eur. J. Inorg. Chem., 2002, 2257.

6. Drain, C. M., Nifiatis, F., Vasenko, A., and Batteas, J. D., Angew. Chem., Int. Ed. Engl., 1998, 37, 2344.

7. Zaworotko, M. J., J. Chem Soc., Chem. Commun., 2001, 1.

8. Roche, S., Haslam, C., Adams, H., Heath, S. L., and Thomas, J. A., J. Chem Soc., Chem. Commun., 1998, 1681.

9. Riese, U., Faza, N., Massa, W., and Dehnicke, K., Angew. Chem., Int. Ed. Engl., 1999, 38, 528.

10. Fujita, M., Ogura, D., Miyazawa, M., Oka, H., Yamaguchi, K., and Ogura, K., Nature, 1995, 378, 469.

11. Hartshorn, C. M. and Steel, P. J., J. Chem Soc., Chem. Commun., 1997, 541.

12. Bourgeois, J.-P. and Fujita, M., Aust. J. Chem., 2002, 55, 619.

13. Ziegler, M., Davis, A. V., Johnson, D. W., and Raymond, K. N., Angew. Chem., Int. Ed., 2003, 42, 665.

14. Olenyuk, B., Levin, M. D., Whiteford, J. A., Shield, J. E., and Stang, P. J., J. Am. Chem. Soc., 1999, $121,10434$.

15. Ternansky, R. J., Balogh, D. W., and Paquette, L. A., J. Am. Chem. Soc., 1982, 104, 4503.

16. For reviews see: Piguet, C., Bernardinelli, G., and Hopfgartner, G., Chem. Rev., 1997, 97, 2005; Albrecht, M., Chem. Rev., 2001, 101, 3457.

17. Wu, A. and Isaacs, L., J. Am. Chem. Soc., 2003, 125, 4831.

18. McMorran, D. A. and Steel, P. J., Angew. Chem., Int. Ed. Engl., 1998, 37, 3295.

19. Sauvage, J.-P., Acc. Chem. Res., 1998, 31, 611.

20. Fujita, M., Acc. Chem. Res., 1999, 32, 53.

This article originally appeared in Chemistry in New Zealand, 2003, 67, pp 57-60, and is reprinted with permission. 